\title{
Phase-Preserving Power Limiting Function Using InP on SOI Photonic Crystal Nanocavity
}

\author{
T. H. Nguyen, K. Lengle, A. Bazin, L. Bramerie, C. Peucheret, M. Gay, O. Sentieys, J. C. Simon, \\ R. Raj and F. Raineri.
}

\begin{abstract}
We report on the use of an InP/SOI hybrid photonic crystal nanocavity for the realization of a phase-preserving alloptical power limiter. This function is experimentally demonstrated with 20-Gbit/s NRZ-QPSK signals. Histogrambased measurements of the phase distributions indicate negligible variations of the phase noise while the amplitude fluctuations are suppressed, confirming the effectiveness of cavity switching for implementing the power-limiter function. Significant power penalty reduction is achieved, indicating a promising application for future photonic circuits.
\end{abstract}

Index Terms - Photonic integrated circuits, photonic crystal nanocavity, power-limiter, optical signal processing.

\section{INTRODUCTION}

A 1l-optical signal processing relies on the availability of nonlinear platforms able to operate at high speed with compact size and low power consumption. For future photonic circuits, InP over silicon-on-insulator (SOI) hybrid technology is an extremely promising solution as it combines CMOS compatibility with the optoelectronic properties of III-V materials. The III-V/SOI hybrid technology has recently allowed the demonstration of lasers [1], amplifiers [2], modulators, flip-flops [3], wavelength converters [4], modulation format converters [5] and 2R-regenerators, including power-limiters [6].

Thanks to their small achievable size with strong optical confinement and enhanced nonlinearity originating from their resonant behavior, photonic crystal (PhC) nanocavities are considered to be promising building blocks for high-density photonic integrated circuits. In particular, all-optical switching has been recently demonstrated using an InP on SOI PhC

Manuscript received $\mathrm{Y} \mathrm{X}^{\text {th }}, 2014$. This work was supported by the European Union (Copernicus project), the Contrat de Plan Etat-Région Ponant and the French Ministry of Research.

We acknowledge I. Sagnes and G. Beaudoin for material growth, R. Braive for participating in the processing of the samples.

T.H. Nguyen, K. Lengle, L. Bramerie, C. Peucheret, M. Gay, J.C. Simon are with Université Européenne de Bretagne (UEB), CNRS-Foton Laboratory (UMR 6082) / Université de Rennes 1, Enssat, BP 80518, 22305 Lannion Cedex, France (e-mail: trung-hien.nguyen@enssat.fr).

O. Sentieys is with INRIA/ IRISA, Campus Beaulieu, 35000 Rennes, France.

A. Bazin, R. Raj, F. Raineri are with Laboratoire de Photonique et de Nanostructures (CNRS UPR 20), route de Nozay, 91460 Marcoussis, France.

F. Raineri is with Universite Paris Diderot, Sorbonne Paris Cité, 75207 Paris Cedex 13, France. nanocavity, and applied to wavelength conversion up to $20 \mathrm{Gbit} / \mathrm{s}$ [7]. However, no detailed analysis of the gate's operational potential as an optical power-limiter for telecommunication signals has been attempted so far.

Over the last decade, there has been a renewed interest for coherent optical communication systems thanks to their improved receiver sensitivity and spectral efficiency, and ability to mitigate transmission impairments in the digital domain [8]. In particular, the quadrature phase-shift-keying (QPSK) format has been the object of intensive investigations and is now employed in commercial 100-Gbit/s transmission solutions. However, the conversion of amplified spontaneous emission (ASE) noise by the transmission fiber Kerr nonlinearity is known to cause nonlinear phase noise (NPN) [9], which may severely degrade QPSK signals, and whose compensation in the digital domain results in increased complexity for the receiver. Instead, amplitude limiters can be used to reduce the accumulation of NPN along the link. It is therefore essential to show that the powerlimiting function we presented in [7] is compatible with QPSK signals with negligible modifications of the phase information.

Phase-preserving amplitude regeneration has been demonstrated using, among others, parametric processes in highly nonlinear fibers [10] or nonlinear amplifying loop mirrors [11,12], which are practically limited by a weak integration capacity. Other techniques using saturable absorbers [13] suffer from high power requirements. As far as $\mathrm{PhCs}$ are concerned, some theoretical studies have been conducted on the power-limiting function [14], without experimental demonstration so far, nor consideration on the phase.

In this letter, a phase-preserving all-optical power-limiter function is experimentally demonstrated by using a $\mathrm{PhC}$ nanocavity based on III-V/SOI hybrid technology. This cavity incorporates a material optimized for ultrafast operation. The limiter performance is investigated with different artificial amplitude noise levels for non return-to-zero (NRZ) QPSK signals at $20 \mathrm{Gbit} / \mathrm{s}$ and estimated in terms of bit error ratio (BER) and phase distribution of the multilevel modulation signal. Improvements of the receiver sensitivity demonstrate the effectiveness of the nanocavity used as a power limiter. Furthermore, amplitude regeneration by nanocavity switching is reported for the first time for a QPSK signal with coherent detection, showing no substantial phase distortion, thus 
demonstrating the compatibility of the scheme with this advanced optical modulation format.

\section{DEVICE DESCRIPTION AND POWER LIMITING PRINCIPLE}
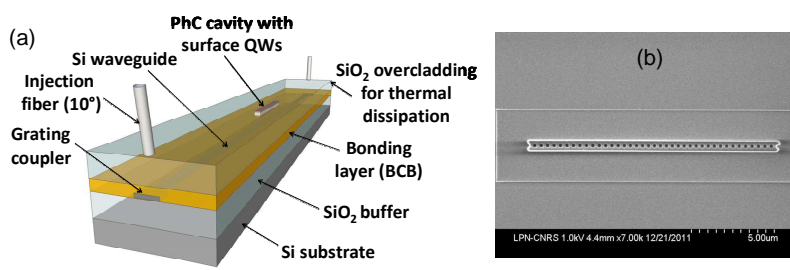

Fig. 1. (a) Device structure and (b) scanning electron microscope picture of the fabricated component.

The passive device, presented in Fig. 1, is composed of two optical levels. The first level consists of a single mode SOI wire waveguide, while the second one is an InP-based $\mathrm{PhC}$ nanocavity [15]. These two levels are separated by a low-index layer (made from silica and benzocyclobutene), which preserves the vertical optical confinement within the SOI waveguide and the $\mathrm{PhC}$ cavity. Optical coupling between the two levels, ensured by the penetration of the evanescent tails of the optical modes into the other level, has been measured to be up to $90 \%$ in a similar structure [1]. The studied nanocavity is a "nanobeam" cavity $[16,17]$, which is formed by drilling holes (targeted radius $r=120 \mathrm{~nm}$, lattice constant around $350 \mathrm{~nm})$ in a single mode InP wire waveguide $(650 \mathrm{~nm} \times 260 \mathrm{~nm} \times 15 \mu \mathrm{m})$ where a single InGaAs quantum well $(8 \mathrm{~nm})$ is grown at both horizontal surfaces. The distance between the holes is subtly varied (around $300 \mathrm{~nm}$ ) [17] in order to obtain a Gaussian apodisation of the electromagnetic field profile. To avoid heating, which usually hampers operation at high bit rates, the cavity is fully encapsulated in $\mathrm{SiO}_{2}$ in order to increase heat evacuation. The SOI waveguide, with an initial width of $400 \mathrm{~nm}$, is tapered up to a width of $10 \mu \mathrm{m}$ so as to etch grating couplers at each extremity to allow coupling with cleaved-facet single-mode optical fibers [18]. Optimal coupling at $1550 \mathrm{~nm}$ is obtained with a fiber angle of $10^{\circ}$. This tapered structure leads to a total device length of $8 \mathrm{~mm}$.

The cavity was first characterized under static operation in order to locate the transmission resonance (Fig. 2(a)). A lowpower continuous wave $(\mathrm{CW})$ signal was coupled into the silicon waveguide via the grating coupler and the transmission was measured as a function of wavelength. The $Q$-factor of the cavity is around 500. The minimum fiber-to-fiber insertion loss is $12 \mathrm{~dB}$ and mainly originates from fiber-to-waveguide coupling loss (25\% estimated coupling efficiency) since the coupling from the SOI waveguide to the InP-based $\mathrm{PhC}$ cavity itself is very efficient [1].

The noise limiter function relies on the self-switching of the cavity under the action of modulated signal intensity. A powerdependent refractive index change shifts the resonance of the cavity towards shorter wavelengths via a change in the carrier density. This frequency shift is schematically represented in Fig. 2(b) to illustrate the principle. If the signal has intensity fluctuations, the dynamic shift will be different depending on the instantaneous intensity of the pulse (shown by the blue or red transmission profiles). The wavelength shift is estimated to be less than $1 \mathrm{~nm}$ under our experimental conditions and enables power limitation, provided that the signal is positioned on the shorter wavelength side of the resonance. The shifted resonance is actually also deeper compared to the initial static resonance emphasizing the transmission loss [7]. By tuning the signal to a suitable wavelength, a transmission function such as the one represented in Fig. 2(c) can be obtained, allowing intensity noise limitation to be performed. Note that, the resonance wavelength can be appropriately tuned with respect to the channel by a suitable thermal control of the device. The response time of the device was evaluated to be as short as 30 ps [19] thanks to the engineered enhancement of nonradiative recombination of carriers, both through surface growth and by patterning the material with dimensions smaller than the carrier diffusion length.

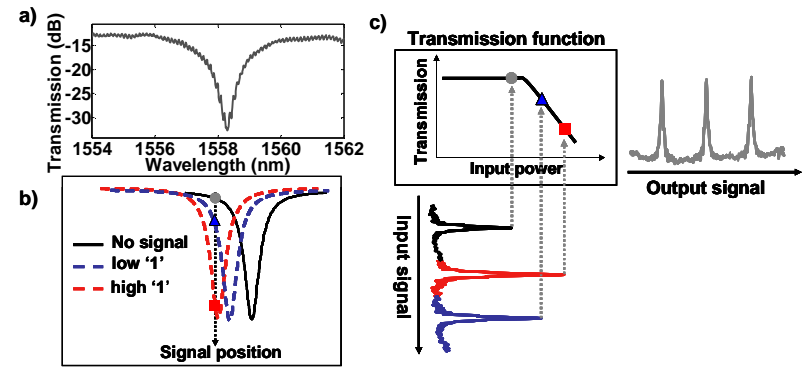

Fig. 2. (a) Measured transmission of the cavity (resolution $0.01 \mathrm{~nm}$ ). (b) Principle of the power limiter function. (c) Transmission function.

\section{SYSTEM ASSESSMENT}

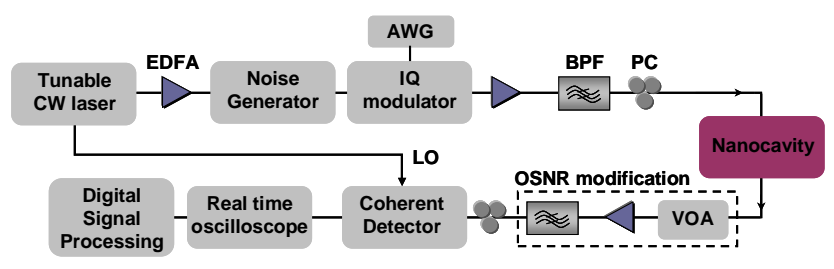

Fig. 3. Experimental setup for power-limitation of a 20-Gbit/s QPSK signal.

The power-limiter function was demonstrated using the implementation of Fig. 3. To evaluate the potential of the device as a power limiter, intensity fluctuations were artificially added to a 10-Gbaud QPSK signal. Intensity noise was generated by externally modulating the output of a CW laser with a pseudo-random binary sequence (PRBS) of length $2^{7}-1$ at $10 \mathrm{Gbit} / \mathrm{s}$. This optical noisy source was then modulated in an IQ modulator driven by two decorrelated PRBSs with lengths of $2^{15}-1$ and $2^{23}-1$ obtained from arbitrary waveform generators (AWGs), resulting in a 10-Gbaud NRZQPSK signal. The noisy modulated signal had its state-ofpolarisation aligned to the TE mode of the input waveguide before being injected into the component at a slightly blue shifted wavelength compared to the cavity resonance. A variable optical attenuator (VOA) followed by an erbiumdoped fiber amplifier (EDFA) and a 3-nm optical bandpass filter (BPF) allowed the adjustment of the optical signal-to- 
noise ratio (OSNR) at the receiver input. An optical $90^{\circ}$ hybrid (100-Gbit/s DP-QPSK integrated receiver) mixed the QPSK signal and the local oscillator (LO). To simplify the setup, a homodyne configuration was employed, with the same laser (linewidth of about $100 \mathrm{kHz}$ ) used at the transmitter and as LO in the receiver. The in-phase and quadrature components were then detected by balanced photodiodes with a bandwidth of $32 \mathrm{GHz}$. Finally, the electrical output signals were measured by a real-time oscilloscope with electrical bandwidth of $16 \mathrm{GHz}$. After data acquisition at $20 \mathrm{GSample} / \mathrm{s}$, postprocessing was performed offline using the Matlab environment.
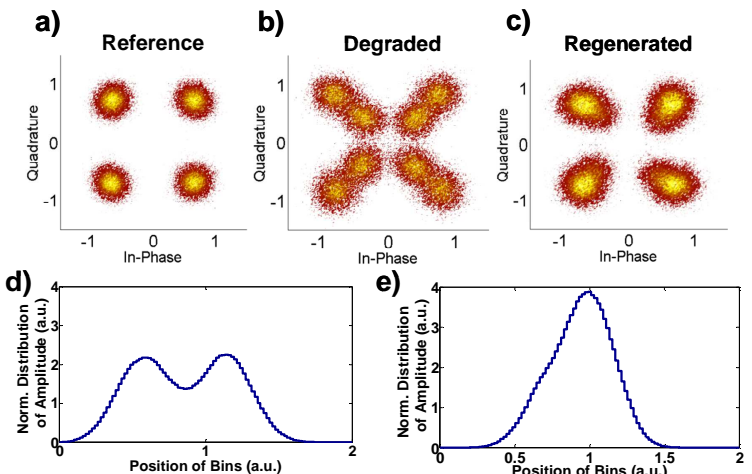

Fig. 4. Experimental constellation diagrams for OSNR at the receiver of

$13 \mathrm{~dB}$ : (a) Reference without additional noise. (b) Degraded signal with additional noise. (c) Regenerated signal through the nanocavity. Amplitude distributions of (d) the degraded signal and (e) the regenerated signal.

Re-timing of the samples was realized with a well-known adaptive finite impulse response (FIR) filter [20] acting as equalizer. After convergence of the feedback loop of the equalizer, the following processes were performed with one sample per symbol after decimating the symbol rate to $10 \mathrm{GSymbol} / \mathrm{s}$. The offline processing was applied to blocks of $2 \times 10^{5}$ samples. The widely-used Viterbi and Viterbi algorithm for carrier phase estimation was performed on blocks of 50 samples, enabling to reconstruct the constellations. Moreover, the phase ambiguity was alleviated by processing the first 5000 samples to minimize the BER. Finally, the decoded bit sequence was compared to the transmitted one to determine the BER over 4 millions samples. Fig. 4 shows examples of constellations of the received QPSK signal for an OSNR of $13 \mathrm{~dB}$ (defined over a 0.1-nm noise bandwidth). Compared to the reference constellation in Fig. 4(a), the effect of the added amplitude fluctuations manifests itself as a splitting of each phase modulation state into two lobes in the constellation diagram, as shown in Fig. 4(b). After passing through the power-limiter, the regenerated constellation (Fig. 4(c)) clearly indicates the effect of intensity noise suppression by merging the split lobes again, even though some residual intensity noise remains compared to the reference constellation.

In order to investigate quantitatively the impact of the noise level, the phase modulation was removed by elevating the processed complex signal samples to the fourth power, so that the phase distributions of all symbols are superimposed at the same angle. The noise magnification induced by the $4^{\text {th }}$ power exponentiation was then compensated by inverse operations (division by four for the phase noise and one-fourth power operation for the amplitude noise). The amplitude and phase distributions of the samples can then be calculated.
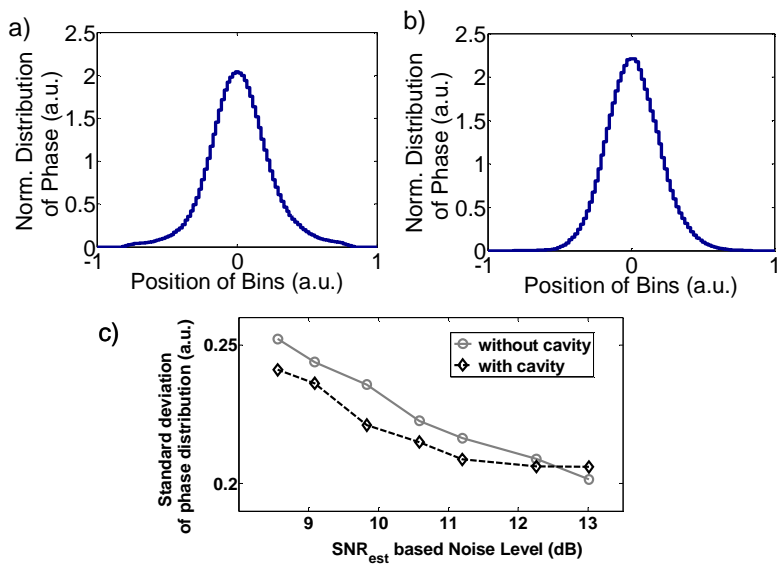

Fig. 5. Impact of the limiter on the phase (for OSNR at the receiver of $13 \mathrm{~dB}$ ). (a) Phase histogram of the degraded signal. (b) Phase histogram of the regenerated signal. (c) Evolution of the standard deviation of the phase with the imposed amplitude noise level.

The amplitude distributions are plotted in Fig. 4(d), in the case of the degraded signal and in Fig. 4(e) in the regenerated case. To be able to evaluate the functionality of the powerlimiter, an estimated SNR, $S N R_{\text {est }}$, is used to quantify the noise level. This parameter is defined as $S N R_{\text {est }}=10 \times \log \left(|\mu|^{2} / \sigma^{2}\right)$, where $\mu$ and $\sigma^{2}$ are the average and variance values of the squared modulus of the processed signal samples. $S N R_{e s t}$ is calculated to be $9.8 \mathrm{~dB}$ before regeneration and $12.2 \mathrm{~dB}$ in the regenerated case, showing an amplitude noise reduction of $2.4 \mathrm{~dB}$.

The impact of the regenerator on the phase of the signal was investigated next for an OSNR at the receiver of $13 \mathrm{~dB}$ and for the same input noise level of $S N R_{\text {est }}=9.8 \mathrm{~dB}$. The phase variation was evaluated by the standard deviation of the phase distribution relatively to the average value. The resulting histograms of the phase distribution obtained before and after regeneration using $2 \times 10^{5}$ symbols are depicted in Fig. 5(a) and (b), respectively. Fig. 5(c) shows the evolution of the phase distribution standard deviation as a function of amplitude SNR based noise level, without regeneration in grey and with regeneration in black. It can be seen that the added amplitude noise level is accompanied with an increase in phase noise variance. However, the standard deviation of the phase noise after the cavity is only marginally lower (about 0.02 at high amplitude noise levels) than that of the unregenerated signal, which proves the phase-preservation of the regenerated signal. The amplitude-to-phase noise transfer induced by the amplitude noise reduction process is therefore very small.

To assess this quality improvement, BER measurements were performed on signals with the same amplitude noise level as in Fig. 5. The theoretical BER curve of a QPSK signal is plotted in Fig. 6 (green diamonds) based on the analysis in [20]. The experimental back-to-back measurement (blue 
stars) matches the theoretical curve with minor differences. First, BER measurements were performed when the transmitter was tuned away from the resonance without additional amplitude noise (red empty circles). This result demonstrates that the transmission through the component does not have any impact on the signal compared to the back-to-back case. Next, the impact of the device away from its resonance on a signal with added amplitude noise was assessed by comparing the resulting BER curve (red filled circles) with that obtained by replacing the device with an attenuator having the same insertion loss (grey filled triangles). Both curves are quite similar, which implies that the effect of the device on the BER performance is negligible away from the resonance. Finally, the measurement was performed on the degraded signal after being switched through the cavity resonance (black cross symbols). This result points out a penalty reduction of up to $6 \mathrm{~dB}$ at a BER of $10^{-3}$ compared to the degraded signal, demonstrating the efficiency of the amplitude noise limiter for phase encoded modulation formats. These results were obtained with a sub-milliwatt coupled peak power.

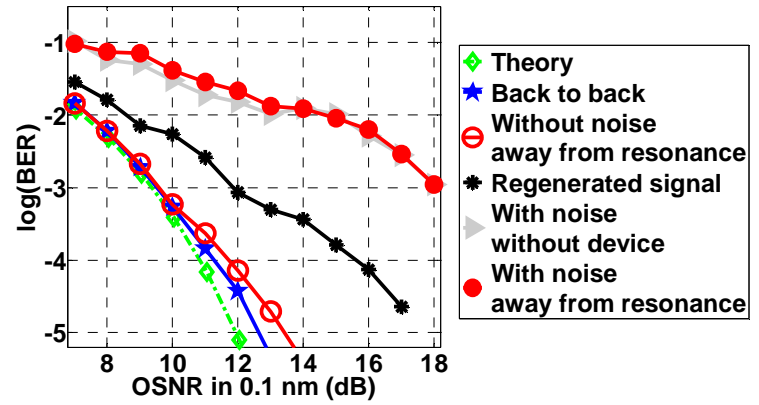

Fig. 6. BER curves of the power-limiter for 20-Gbit/s QPSK (for artificial amplitude noise level $S N R_{\text {est }}=9.8 \mathrm{~dB}$ ).

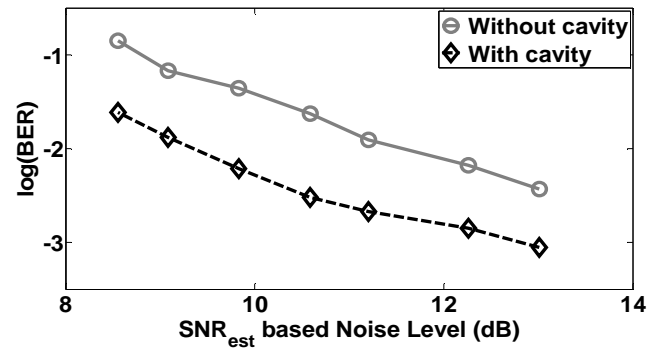

Fig. 7. Evolution of the BER as a function of the amplitude noise level for a 20-Gbit/s QPSK signal with and without power limitation (for an OSNR at the receiver of $10 \mathrm{~dB}$ ).

Finally, the efficiency of the device was investigated as a function of amplitude noise level. BER measurements were performed for different artificial amplitude noise levels and a received OSNR of $10 \mathrm{~dB}$, as shown in Fig. 7, with (black diamond), and without (grey circle) the device. The BER is severely degraded at high-amplitude noise levels compared to low noise levels. However it can be noticed that, over the studied noise level range, the BER is improved by one order of magnitude after the cavity and that the improvement remains constant regardless of the input amplitude noise level. At a noise level $S N R_{\text {est }}=13 \mathrm{~dB}$, performance similar to the back-toback case is obtained after regeneration, confirming the effectiveness of the power-limiter.

\section{CONCLUSION}

In this letter, an experimental analysis of the power-limiting properties of an optical gate based on a hybrid $\mathrm{InP} \mathrm{PhC}$ nanocavity on an SOI substrate is reported. Effective intensity noise reduction was obtained for 20-Gbit/s NRZ-QPSK signals. The phase preserving capability of the power-limiter was demonstrated, with up to $6 \mathrm{~dB}$ power penalty reduction at a BER of $10^{-3}$.

\section{REFERENCES}

[1] Y. Halioua et al., "Hybrid III-V semiconductor/silicon nanolaser," Opt. Express, vol. 19, no. 10, pp. 9221-9231, May 2011.

[2] H. Park et al., "A hybrid AlGaInAs-silicon evanescent amplifier," IEEE Photon. Technol. Lett., vol. 19, no. 4, pp. 230-232, Feb. 2007.

[3] D. Van Thourhout et al., "Nanophotonic devices for optical interconnect," IEEE J. Sel. Topics Quantum Electron., vol. 16, no. 5, pp. 1363-1375, Sep./Oct. 2010.

[4] R. Kumar et al., "Ultrafast and bias-free all-optical wavelength conversion using III-V-on-silicon technology," Opt. Lett., vol. 36, no. 13 , pp. 2450-2452, Jul. 2011.

[5] R. Kumar et al., "10 Gbit/s all-optical NRZ-OOK to RZ-OOK format conversion in an ultra-small III-V-on silicon microdisk fabricated in a CMOS pilot line," Opt. Express, vol. 19, no. 10, pp. 24647-24656, Nov. 2011

[6] P. Mechet et al., "All-optical low-power $2 \mathrm{R}$ regeneration of $10 \mathrm{~Gb} / \mathrm{s}$ NRZ signals using a III-V on SOI microdisk laser," IEEE Photon. J., vol. 5, no. 6, 7802510, Dec. 2013.

[7] K. Lengle et al., "Modulation contrast optimization for wavelength conversion of a $20 \mathrm{Gbit} / \mathrm{s}$ data signal in hybrid InP/SOI photonic crystal nanocavity", Opt. Lett., vol. 39, no. 9, 2014 (to be published).

[8] E. Ip et al., "Coherent detection in optical fiber systems," Opt. Express vol. 16, no. 2, pp. 753-791, Jan. 2008.

[9] J. P. Gordon et al., "Phase noise in photonic communication systems using linear amplifiers," Opt. Lett., vol. 15, no. 23, pp.1351-1354, Dec. 1990.

[10] M. Matsumoto et al., "Performance improvement of DPSK signal transmission by a phase-preserving amplitude limiter," Opt. Express, vol. 15 , no. 13 , pp. 8094-8103, Jun. 2007.

[11] S. Boscolo et al., "Design of Raman-based nonlinear loop mirror for alloptical 2R regeneration of differential phase-shift-keying transmission," IEEE J. Quantum Electron., vol. 42, no. 7, pp. 619-624, Jul. 2006.

[12] C. Stephan et al., "Phase-preserving amplitude regeneration in DPSK transmission systems using a nonlinear amplifying loop mirror," IEEE J. Quantum Electron., vol. 45, no. 11, pp. 1336-1343, Nov. 2009.

[13] L. Bramerie et al., "All-optical 2R regeneration with a vertical microcavity-based saturable absorber," IEEE J. Sel. Topics Quantum Electron., vol. 18, no. 2, pp. 870-883, Mar./Apr. 2012.

[14] M. Bahl et al., "Nonlinear optical effects in a two-dimensional photonic crystal containing one-dimensional Kerr defects," Phys. Rev. E, vol. 67,no. 5, 056604, May 2003.

[15] T. J. Karle et al., "Heterogeneous integration and precise alignment of InP-based photonic crystal lasers to complementary metal-oxide semiconductor fabricated silicon-on-insulator wire waveguides" J. App. Phys., vol. 107, no. 6,063103, Mar.2010.

[16] J. S. Foresi et al., "Photonic-bandgap microcavities in optical waveguides," Nature, vol. 390, no. 6656, pp. 143-145, Nov. 1997.

[17] A. Bazin et al., "Design of silica encapsulated high-Q photonic crystal nanobeam cavity", IEEE J. Lightwave Technol., vol. 32, no. 5, pp. 952958, 2014.

[18] D. Taillaert et al., "Grating couplers for coupling between optical fibers and nanophotonic waveguides," Jpn. J. Appl. Phys., vol. 45, no. 8A, pp. 6071-6077, Aug. 2006

[19] A. Bazin, et al., "Ultrafast all-optical switching and error-free $10 \mathrm{Gbit} / \mathrm{s}$ wavelength conversion in hybrid InP-silicon on insulator nanocavities using surface quantum wells", Appl. Phys. Lett. 104, 011102, 2014.

[20] J. Proakis, Digital Communications, (McGraw-Hill, $4^{\text {th }}$ edition, 2000), Chap. 5 and Chap. 11. 